\title{
Metodología para la Enseñanza del Movimiento Oscilatorio mediante Simulación Computarizada
}

\author{
Héctor F. Collazos y Omar D. Castrillón \\ Universidad Nacional de Colombia - Sede Manizales, Facultad de Ingeniería y Arquitectura, \\ Departamento de Ingeniería Industrial, Grupo de Innovación y Desarrollo Tecnológico, Bloque Q Campus La Nubia, \\ Manizales, 170001 - Colombia. (e-mail: hfcollazosv@unal.edu.co, odcastrillong@unal.edu.co)
}

Recibido Oct. 16, 2018; Aceptado Dic. 27, 2018; Versión final Feb. 11, 2019, Publicado Ago. 2019

\section{Resumen}

Se diseñó una metodología de enseñanza utilizando simulaciones computarizadas para analizar su impacto sobre el nivel de satisfacción de los estudiantes con la enseñanza de fenómenos físicos. Se crearon cinco simulaciones sobre movimiento oscilatorio empleando el software GeoGebra, y se creó un cuestionario para motivar la exploración autónoma de las simulaciones. En el grupo control se orientaron los temas de forma tradicional y en el grupo experimental se combinó la metodología tradicional con el uso de las simulaciones. Se aplicó un test de Likert para medir el nivel de satisfacción de manera previa y posterior a la aplicación de la metodología. En las respuestas al test de Likert, se presentó una variación porcentual del nivel de satisfacción que para el grupo control oscila entre $-5,0 \%$ y $3,6 \%$, y para el grupo experimental entre $1,3 \%$ y $12 \%$. Basado en los resultados se concluye que la metodología asistida por simulaciones computarizadas permite obtener una variación positiva en el nivel de satisfacción, mayor que con la metodología tradicional.

\section{Methodology for the Teaching of Oscillatory Motion using Computer Simulation}

\begin{abstract}
A teaching methodology was designed using computer simulation to analyze its impact on student's satisfaction level with the teaching of physical phenomena. Five simulations on oscillatory movement were created using the GeoGebra software, and a questionnaire was designed to motivate the autonomous exploration of the simulations. In the control group the subjects were oriented in a traditional way and in the experimental group the traditional methodology was combined with the use of the simulations. A Likert test was applied to measure the satisfaction level before and after the application of the methodology. In the responses to the Likert test, a percentage variation of the level of satisfaction was presented, which for the control group ranges between $-5.0 \%$ and $3.6 \%$, and for the experimental group between $1.3 \%$ and $12 \%$. Based on the results it is concluded that the methodology assisted by computer simulations allows to obtain a positive variation in the satisfaction level, greater than that which uses the traditional methodology.
\end{abstract}




\section{INTRODUCCIÓN}

La labor pedagógica de los docentes actualmente se ve enfrentada a la problemática de la búsqueda de herramientas que permitan mejorar la motivación en el aprendizaje de los estudiantes (Díez, 2015), quienes se observan con menos empeño ante el aprendizaje a través de los métodos de enseñanza tradicionales. De acuerdo a lo anterior y teniendo en cuenta la posición actual que ocupan las nuevas tecnologías en la sociedad y en particular en la vida de los jóvenes, se hace necesario incorporarlas al sistema educativo vigente (Smetana y Bell, 2012), para de esta manera lograr una transformación del enfoque de enseñanza actual (Huapaya et al., 2005) hacia un sistema que sea fácilmente asimilado por cualquier estudiante, independiente de sus características. En el área de ciencias naturales se genera un gran interés por la investigación de nuevos métodos de enseñanza (Cardona y López, 2017), debido a que es una de las áreas donde se observan de forma más evidente las problemáticas en el aprendizaje (Dinescu et al., 2010), sobresaliendo fundamentalmente la dificultad en la enseñanza de la física, una de las materias con mayores obstáculos en su comprensión (Rubio, 2013). Generalmente la enseñanza se basa en sistemas tradicionales donde el alumno es sólo un receptor pasivo de conocimiento (Zuluaga, 2016), además en estos modelos se pretende transmitir los conocimientos de la misma manera a todos los alumnos, sin considerar la importancia de las características de personalidad, ritmo de aprendizaje, o prácticas de estudio particulares, lo cual puede limitar el nivel de aprendizaje (Huapaya et al., 2005), generando en muchos casos que el estudiante solo aprenda momentáneamente, se desmotive y por tanto no interiorice o reconozca la importancia aplicada a la realidad del aprendizaje que está adquiriendo, el cual se busca sea perdurable en el tiempo (Díez, 2015).

El ideal es identificar la combinación de estrategias de enseñanza tanto tradicionales como interactivas (libros, videos, simulaciones, etc.), que permitan generar en el estudiante un mayor interés y estimular su participación en clase (Márquez y Cárdenas, 2008) y por tanto mejorar la interpretación a través del aprendizaje teórico, práctico y experimental, de forma que los alumnos tomen el control de su formación, incrementen su interés para poder relacionar los conocimientos adquiridos con situaciones de la vida cotidiana y de esta forma mejorar la comprensión de lo aprendido. (Zacharia, 2007). La enseñanza de las ciencias a partir de metodologías no tradicionales, usando herramientas didácticas, ha sido objeto de varias investigaciones, las cuales se han enfocado en la implementación de herramientas tecnológicas, algunas basadas en simulaciones por computadora, otras en prácticas experimentales (Zacharia, 2007; Chang et al., 2008; Dinescu et al., 2010; Stoica et al., 2011; Smetana y Bell, 2012; Krobthong, 2015; Carpenter et al., 2016). La generalidad de los estudios demuestra la necesidad de la incorporación de nuevas tecnologías, como por ejemplo software especializado, en las aulas y otros ambientes laborales de la actualidad, ya que estas herramientas pueden contribuir a que los estudiantes logren una mayor comprensión y aprendan más sobre ciencia (Rossi y Baldini, 2008; Zucker y Hug, 2008) y su posición frente a ella cambie favorablemente y por tanto se pueda contribuir con la mejora en la calidad educativa (Summak et al., 2010).

Algunos autores afirman que una metodología efectiva debe favorecer los cambios conceptuales en el entorno de aprendizaje (Zacharia, 2007), logrando un impacto positivo en la actitud del estudiante hacia el aprendizaje de la física, potenciando sus habilidades (Jaramillo, 2016) y desarrollando la motivación intrínseca, contando de esta forma con estudiantes motivados por aprender tanto en el aula como fuera de ésta (Díez, 2015). Además, las simulaciones aplicadas en la enseñanza de la física pueden contribuir a la interacción y comunicación bidireccional entre estudiantes y docentes, despertando de esta forma un mayor interés en la materia (Dega et al., 2013). En la presente investigación, a partir de la implementación de una metodología de enseñanza asistida por simulaciones computarizadas, se pretende cuantificar el cambio en el nivel de satisfacción hacia el aprendizaje de la física. Se busca promover la exploración autónoma de las simulaciones a través de unas preguntas guía que se proporcionan mediante un cuestionario de apoyo.

\section{MATERIALES Y MÉTODOS}

La población estudiada corresponde a estudiantes de grado undécimo de la Institución Educativa La Sagrada Familia del municipio de Palestina, en el departamento Caldas, Colombia. La institución es de carácter mixto y pertenece al sector oficial. Los contenidos de la asignatura física se orientan de acuerdo a los lineamientos curriculares del Ministerio de Educación Nacional de Colombia. El estudio se realizó con 59 estudiantes, la muestra está conformada por 32 mujeres y 27 hombres en edades comprendidas entre los 17 y los 19 años. Se seleccionaron dos grupos de estudio, el primero llamado grupo control (11-1) está conformado por 30 estudiantes y el segundo llamado grupo experimental (11-2) constituido por 29 estudiantes, ambos grupos son de carácter mixto. 


\section{Paso 1. Diseño de las simulaciones:}

Existe gran variedad de software tanto de pago como gratuito que se enfoca en la simulación de fenómenos físicos. Desde aplicaciones comerciales hasta software de uso libre bajo diferentes acuerdos de licencia, entre los más populares se encuentra el proyecto Phet de la universidad del colorado, que presenta un conjunto de simulaciones programadas a través de diversos lenguajes, y que cubren diferentes áreas de las ciencias y matemáticas. Las simulaciones del proyecto Phet se encuentran en continuo desarrollo, son amigables con el usuario y están disponibles para descarga libre (Krobthong, 2015).

La interacción del usuario con una simulación tiene un carácter exploratorio, mientras que en una modelación el usuario tiene acceso a la estructura básica y está en capacidad de construir el modelo desde cero, modificarlo o reconstruirlo conforme desee (Lopez et al., 2016). En este sentido la mayor parte del software disponible sobre fenómenos físicos incluido el proyecto Phet, funcionan como simuladores. Para esta investigación se seleccionó geogebra, un software gratuito empleado para enseñar y aprender matemáticas, permite a sus usuarios crear modelos e interactuar con ellos, geogebra acepta comandos relacionados con geometría, álgebra y cálculo; posibilita la aplicación interactiva de diferentes temáticas, presentándolas en forma de animaciones y permitiendo una exploración visual y dinámica (Caligaris et al., 2015). Esta herramienta permite la familiarización del estudiante con el tema de estudio, a través de la visualización de los resultados de mediciones realizadas (León, 2017). Se diseñaron en total 5 simulaciones las cuales se describen en la Tabla 1 a través de los algoritmos básicos utilizados para su construcción.

Tabla 1: algoritmos utilizados para construir las simulaciones en geogebra.

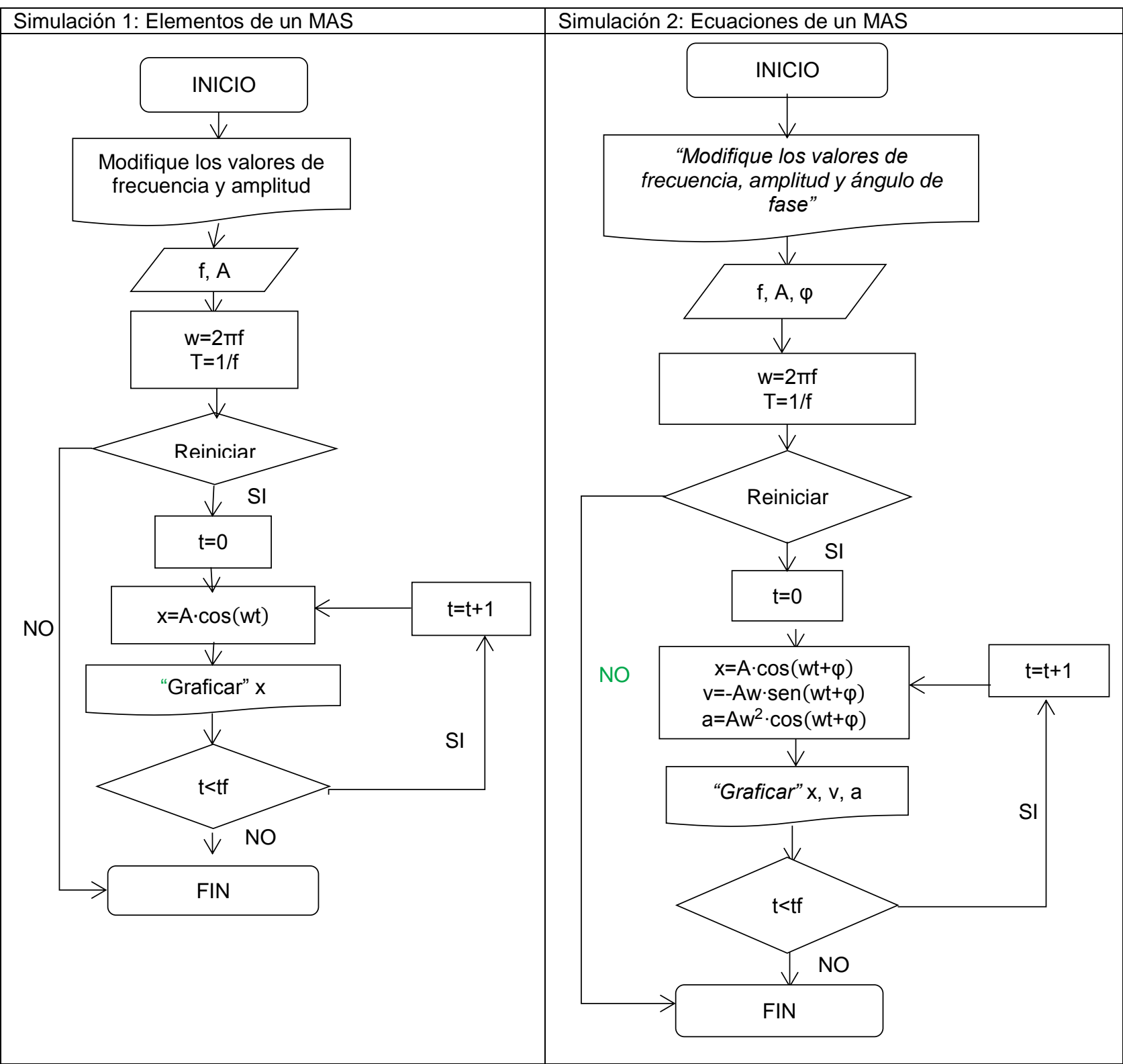


Tabla 1 (continuación)

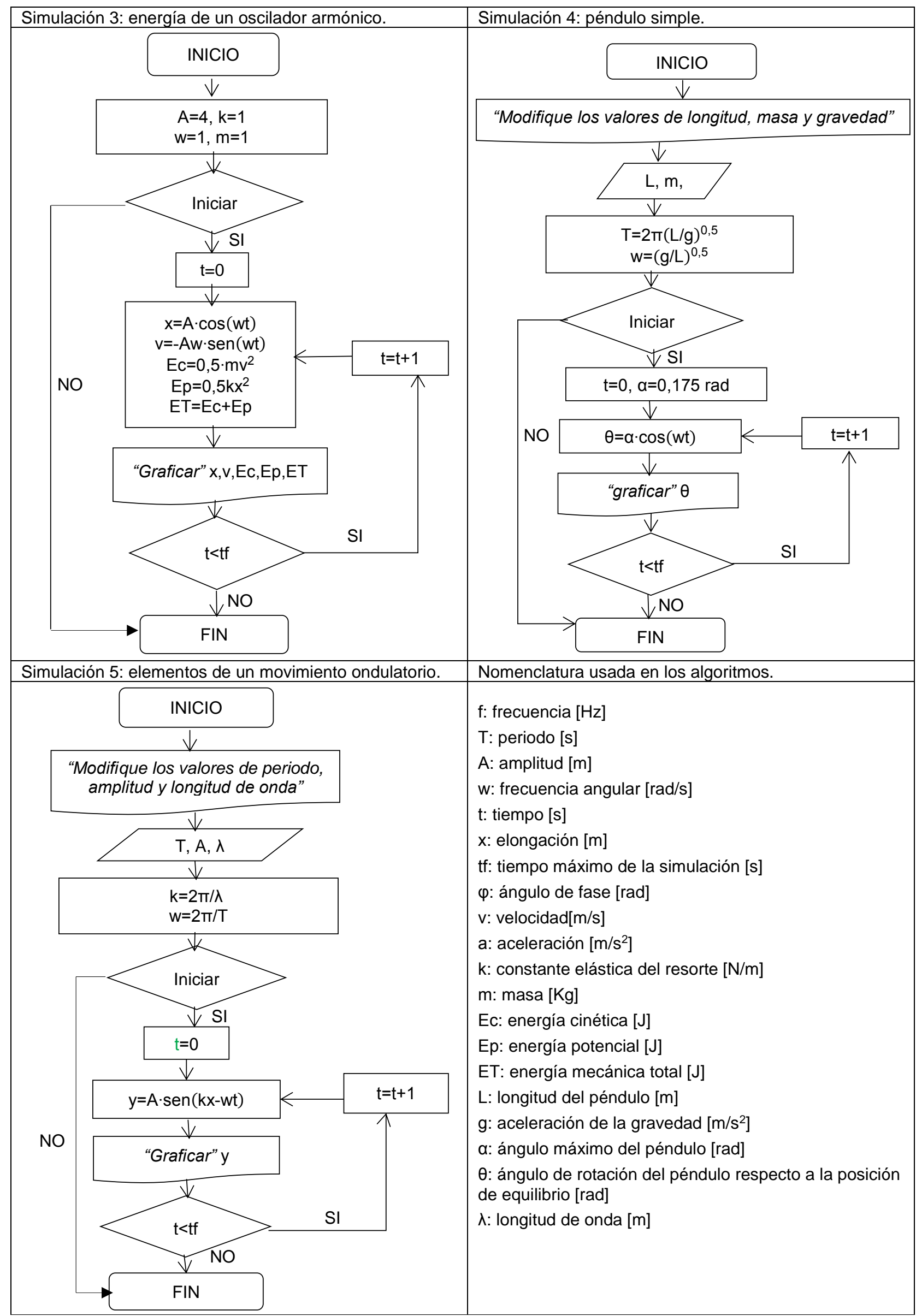


La mayoría de las investigaciones orientadas a la implementación de software en las aulas de clase de física se enfocan en la utilización de simulaciones, poca utilización se da a las actividades de modelación computacional, probablemente debido a que implica aparte del conocimiento de los modelos matemáticos conocimientos en la utilización de un lenguaje de programación especifico (Lopez et al., 2016). En este sentido la investigación que se presenta en este artículo contiene elementos de modelación dado que los escenarios de los fenómenos estudiados fueron construidos desde cero a partir de sus modelos matemáticos, sin embargo, el producto de estos modelos computacionales se presenta al estudiante como una serie de simulaciones, en las cuales debe manipular variables y observar el comportamiento presentado.

\section{Paso 2. Diseño de los cuestionarios de apoyo.}

Para guiar el trabajo con cada una de las simulaciones se generaron una serie de preguntas con el fin de motivar la exploración por parte del estudiante de la relación existente entre las diferentes variables, las preguntas se muestran en la Tabla 2.

Tabla 2: preguntas realizadas en los cuestionarios de apoyo.0

\section{Simulación 1. Elementos de un MAS}

1. ¿Qué efecto tiene modificar la frecuencia de oscilación del sistema masa-resorte sobre la gráfica trazada en el papel milimetrado?

2. ¿Qué efecto tiene modificar la amplitud de oscilación del sistema masa resorte sobre la gráfica trazada en el papel milimetrado?

3. ¿Cómo se modifica la velocidad del sistema masa resorte al variar la frecuencia?

4. ¿Cómo se modifica el periodo de oscilación al variar la frecuencia?

5. ¿Qué relación existe entre frecuencia y amplitud?

Simulación 3. Energía en un oscilador armónico

1. ¿De qué depende la energía cinética del sistema?

2. ¿De qué depende la energía potencial del sistema?

3. ¿Por qué la energía mecánica no cambia?

4. ¿En qué puntos de la trayectoria la energía cinética es máxima y en qué puntos vale cero?

5. ¿En qué puntos de la trayectoria la energía potencial es máxima y en qué puntos vale cero?
Simulación 2. Ecuaciones de un MAS

1. ¿Qué sucede al variar el ángulo phi?

2. ¿En qué puntos de la trayectoria la velocidad vale cero?

3. ¿En qué puntos de la trayectoria la velocidad es máxima?

4. ¿Cómo se interpreta la velocidad negativa?

5. ¿En qué puntos de la trayectoria la aceleración vale cero?

6. ¿En qué puntos de la trayectoria la aceleración es máxima?

Simulación 4. Péndulo simple

1. ¿Qué sucede con el periodo del péndulo al variar la longitud de la cuerda?

2. ¿Qué sucede con el periodo del péndulo al variar la aceleración de la gravedad?

3. ¿Qué sucede con el periodo del péndulo al variar la masa del péndulo?

Simulación 5. Elementos de una onda

1. ¿Qué sucede con la animación al variar el periodo?

2. ¿Qué sucede con la animación al variar la amplitud?

3. ¿Qué sucede con la animación al variar la longitud de onda?

Paso 3. Diseño del test de Likert:

Una forma de abordar la investigación en el aprendizaje interactivo es a través de la aplicación de la herramienta test de Likert, una escala que permite hacer una medición imparcial (Hartley, 2013) y que está constituida por una serie de preguntas que pretenden conocer la actitud del encuestado frente al tema de referencia (Harpe, 2015), la encuesta muestra una serie de opciones de respuesta que indican si el encuestado está o no de acuerdo con el enunciado que se le propone, mostrando una posición frente al tema objeto del estudio (Jaramillo, 2016). La escala de Likert puede ser aplicada antes y después de la implementación de los cambios específicos para determinar e identificar de esta forma la percepción de los estudiantes frente a la metodología implementada y por tanto la favorabilidad de la misma, además de medir el grado de satisfacción de los estudiantes con el modelo de enseñanza aplicado (Harpe, 2015).

Para el presente trabajo se desarrolló un test conformado por 10 preguntas, de las cuales 5 son favorables, es decir los enunciados están a favor del estímulo o la actitud del estudiante y 5 son desfavorables, es decir están en contra del estímulo (Delgadillo, 2013). Las opciones de respuesta para cada una de ellas fueron: Total Desacuerdo, En Desacuerdo, Indeciso, De acuerdo y Totalmente de acuerdo. Con las preguntas se pretende identificar el grado de satisfacción de los estudiantes frente al uso de los recursos y metodologías aplicadas en la asignatura y su percepción frente a la física. Además, que tan efectivo es este método de enseñanza y si a partir 
de él se pueden generar cambios de actitud de los estudiantes frente al aprendizaje de la física. En la Tabla 3 se muestra la versión final de las preguntas incorporadas en el test.

La escala de valoración para cada pregunta se asignó de 1 a 5 , siendo 1 totalmente en desacuerdo y 5 totalmente de acuerdo para los ítems favorables y la escala inversa para las preguntas desfavorables (Delgadillo, 2013), tal como se muestra en la Tabla 4.

Tabla 3: ítems del test de Likert aplicado a grupos experimental y de control.

\begin{tabular}{|c|l|l|}
\hline No. & \multicolumn{1}{|c|}{ Pregunta } & Intención \\
\hline 1 & $\begin{array}{l}\text { Las herramientas utilizadas en la asignatura me permiten entender conceptos metodológicos } \\
\text { complicados. }\end{array}$ & Favorable \\
\hline 2 & $\begin{array}{l}\text { La metodología de enseñanza de la asignatura no me permite explorar por mi propia cuenta el } \\
\text { comportamiento de los sistemas físicos estudiados. }\end{array}$ & Desfavorable \\
\hline 3 & $\begin{array}{l}\text { Considero que la intensidad horaria de la asignatura se distribuye apropiadamente entre la teoría } \\
\text { y la práctica }\end{array}$ & Favorable \\
\hline 4 & Al iniciar un tema nuevo no me preguntan qué conocimiento tengo acerca de él. & Desfavorable \\
\hline 5 & $\begin{array}{l}\text { Prefiero aprender a través de contenidos multimedia que con las clases tradicionales de tablero } \\
\text { y marcador. }\end{array}$ & Favorable \\
\hline 6 & $\begin{array}{l}\text { Considero que los temas estudiados no tienen aplicación práctica. } \\
\text { asignatura. }\end{array}$ & $\begin{array}{l}\text { Me gustaría que otros docentes empleen herramientas similares a las utilizadas en esta } \\
\text { aprable }\end{array}$ \\
\hline 8 & $\begin{array}{l}\text { Fl docente se interesa por enseñar todos los temas de la asignatura, más que por alcanzar el } \\
\text { aprendizaje de sus estudiantes. }\end{array}$ & Desfavorable \\
\hline 9 & El docente alienta la participación y la discusión acerca de los temas estudiados en clase. & Favorable \\
\hline 10 & $\begin{array}{l}\text { En la asignatura no se optimiza la utilización de recursos tecnológicos para la enseñanza } \\
\text { (Computadores, tabletas, proyectores, internet, etc). }\end{array}$ & Desfavorable \\
\hline
\end{tabular}

Tabla 4: Escala de valoración para los ítems del test de Likert.

\begin{tabular}{|l|c|c|}
\hline \multirow{2}{*}{ Alternativas } & Ítems favorables & Ítems desfavorables \\
\cline { 2 - 3 } & Valor & Valor \\
\hline Total Desacuerdo & 1 & 5 \\
\hline En desacuerdo & 2 & 4 \\
\hline Indeciso & 3 & 3 \\
\hline De acuerdo & 4 & 2 \\
\hline Totalmente de acuerdo & 5 & 1 \\
\hline
\end{tabular}

Paso 4. Aplicación de la metodología:

Para ambos grupos se aplicó el test de Likert al inicio del tercer periodo académico, con el fin de medir el nivel de satisfacción hacia la metodología tradicional de enseñanza. Para los dos grupos se desarrolló el mismo plan de estudios, es decir, se orientaron los mismos temas correspondientes a movimiento armónico simple y movimiento ondulatorio. En el grupo de control se emplearon clases teóricas, solución de problemas y evaluación escrita, en el grupo experimental además de lo anterior se realizaron sesiones adicionales empleando las simulaciones computarizadas. Cada estudiante del grupo experimental tuvo acceso a un computador portátil para correr las simulaciones y a una versión impresa del cuestionario de apoyo. Una vez culminado el tercer periodo académico se aplicó nuevamente el test de Likert en ambos grupos, con el fin de conocer la influencia de las metodologías aplicadas en el nivel de satisfacción de los estudiantes.

\section{RESULTADOS}

A continuación, se presentan los resultados para cada uno de los pasos de la metodología:

\section{Paso 1. Simulaciones.}

Simulación 1. Elementos de un MAS: se presenta un objeto unido a un resorte, el sistema oscila de forma vertical y su movimiento es registrado sobre un papel cuadriculado que se desplaza de forma horizontal. Se pueden modificar la frecuencia y la amplitud de la oscilación. El tiempo de la animación está fijado a 20 seg., los cuales se pueden visualizar en el cronometro. En cualquier instante de la animación se pueden variar los valores de frecuencia y amplitud y observar el resultado sobre el trazo generado en el papel (Fig. 1.) 


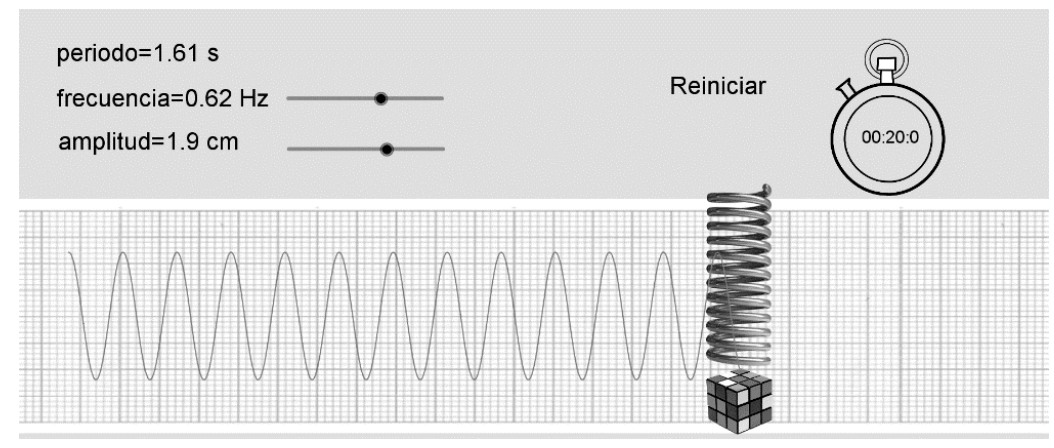

Fig. 1: interfaz gráfica de la simulación "elementos de un MAS".

Simulación 2. Ecuaciones de un MAS: se utiliza el mismo sistema masa-resorte de la simulación 1. El resorte empieza a oscilar desde su posición inicial determinada por el ángulo phi. Al lado derecho de la simulación se van trazando las gráficas de posición, velocidad y aceleración en función del tiempo, dichas gráficas se pueden mostrar $u$ ocultar utilizando las casillas de verificación. El botón $t \rightarrow 0$ reinicia el deslizador de tiempo y hace visible el deslizador del ángulo phi lo cual permite variar la posición inicial del resorte. En la Figura 2 se muestra una captura de pantalla de la simulación 2.

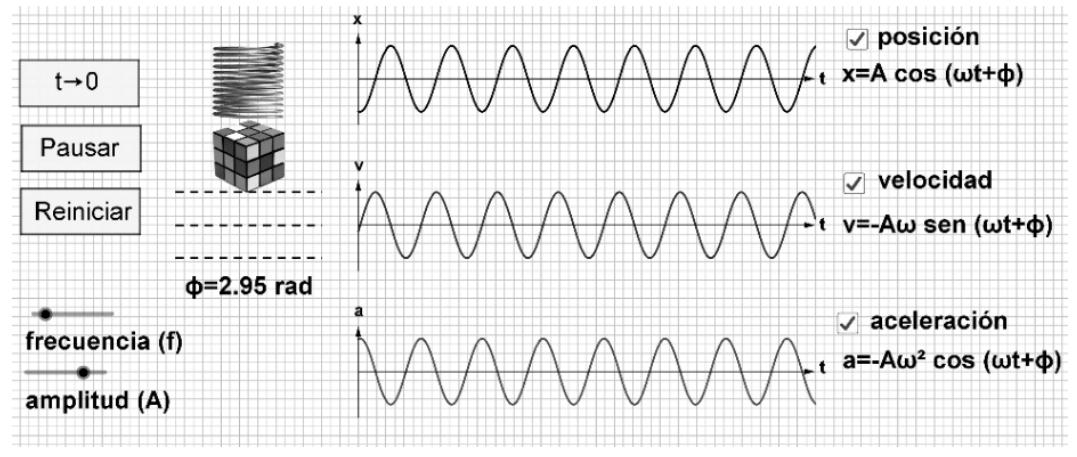

Fig. 2: Interfaz gráfica de la simulación ecuaciones de un MAS.

Simulación 3. Energía de un oscilador armónico: se utilizan dos ventanas gráficas, en la ventana de la izquierda se muestra mediante un diagrama de barras como varían la energía potencial, la energía cinética y la energía mecánica total del sistema masa-resorte, de acuerdo a la posición. En la ventana de la derecha se muestra una gráfica de energía potencial elástica en función de la elongación del resorte para el mismo sistema. En esta simulación el usuario no puede modificar ninguna variable, simplemente su rol se limita a observar como varia la energía del sistema de acuerdo a la posición. La Figura 3 muestra una captura de pantalla de la simulación 3.

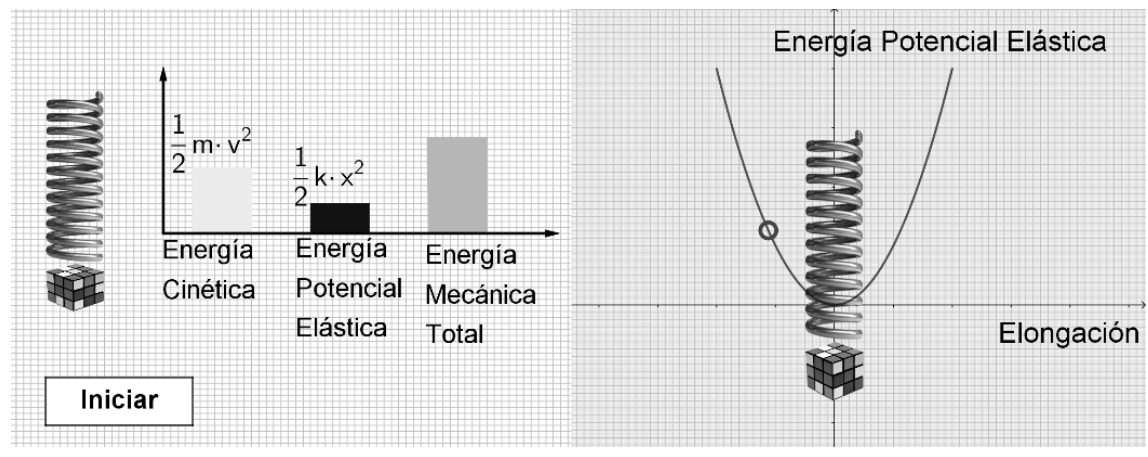

Fig. 3: Interfaz gráfica de la simulación energía de un oscilador armónico simple.

Simulación 4. Péndulo simple: se presenta un modelo de un péndulo simple. Existen tres deslizadores que permiten variar la longitud del péndulo, la aceleración de la gravedad y la masa del péndulo. En pantalla se presenta información sobre la fórmula para calcular el periodo, el tiempo de oscilación y el periodo de oscilación. La Figura 4 muestra una captura de pantalla de la simulación 4. 


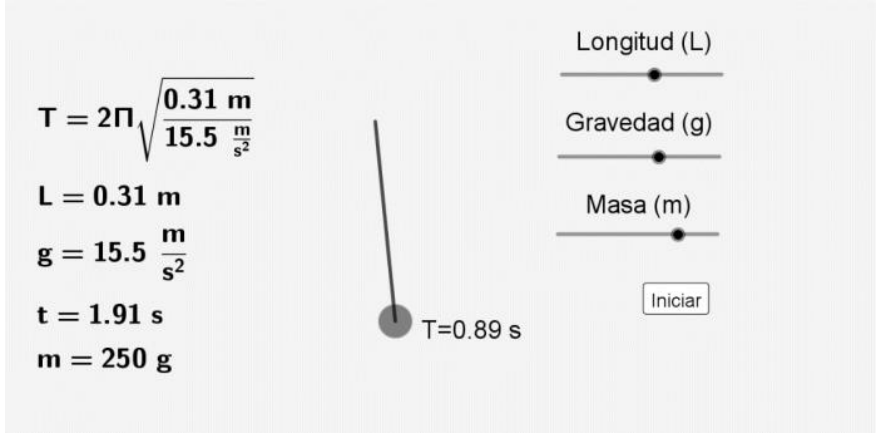

Fig. 4: Interfaz gráfica de la simulación péndulo simple.

Simulación 5. Elementos de una onda: en esta simulación se pueden manipular los valores de periodo, amplitud y longitud de onda mediante el uso de deslizadores. De igual forma se puede analizar por separado el efecto de cada una de estas tres variables, utilizando las casillas de verificación correspondientes, como se muestra en la Figura 5.

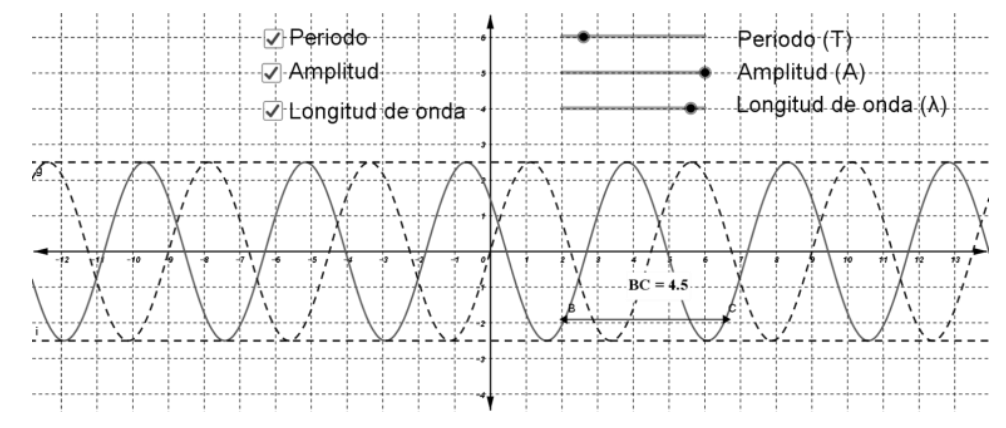

Fig. 5: Interfaz gráfica de la simulación elementos de una onda.

\section{Paso 2. Cuestionario de apoyo.}

El cuestionario de apoyo presentado en la Tabla 2 contiene preguntas de carácter abierto, por consiguiente, las respuestas dadas por los estudiantes a este cuestionario son muy variadas, se optó por agrupar las respuestas a partir de conceptos claves. Dichos conceptos permiten inferir el nivel de comprensión que tienen los estudiantes hacia el tema tratado. En la Tabla 5 se muestra un resumen de las respuestas obtenidas al aplicar el cuestionario de apoyo al grupo experimental. Las respuestas que presentaron baja frecuencia (menos de 3 repeticiones) se agruparon bajo la denominación otras respuestas.

En muchas de las respuestas se evidencia una correcta asociación entre el comportamiento del fenómeno estudiado y las variables manipuladas, por ejemplo para la primera pregunta de la simulación 1: ¿Qué efecto tiene modificar la frecuencia de oscilación del sistema masa-resorte sobre la gráfica trazada en el papel milimetrado?, en el $44,8 \%$ de las respuestas obtenidas los estudiantes manifiestan que cambia la velocidad con que se mueve el sistema masa-resorte; al ser la frecuencia una medida del número de oscilaciones por segundo que alcanza el sistema oscilante, existe una relación directa entre la frecuencia y la velocidad con que se mueve el sistema masa-resorte, como lo manifiestan el $20,7 \%$ de los estudiantes al responder que a mayor frecuencia mayor velocidad y viceversa. En el 17,2 \% de las respuestas los estudiantes manifiestan que "el trazado se comprime o expande horizontalmente" como efectivamente ocurre, mientras tanto el 10,3 $\%$ de los estudiantes manifiesta que el periodo cambia, lo cual es cierto por la relación inversa que existe entre las variables periodo y frecuencia y además indica atención a los detalles por parte de los estudiantes que observaron el cambio en el valor numérico de dicho parámetro que se muestra en la interfaz gráfica de la simulación.

Sin embargo, en algunas de las respuestas se evidencia confusión de conceptos, por ejemplo: en la pregunta 2 de la simulación 1 ¿Qué efecto tiene modificar la amplitud de oscilación del sistema masa-resorte sobre la gráfica trazada en el papel milimetrado? El 20,7 \% de los estudiantes respondieron "cambia el tamaño de la onda" utilizando erróneamente el término onda en lugar de oscilación, de igual manera en la pregunta 5 de la simulación 1, ¿Qué relación existe entre frecuencia y amplitud? El 17,2 \% responde "frecuencia es la cantidad de ondas por minuto, amplitud es la carga energética." Intercambiando nuevamente los conceptos de onda y oscilación y utilizando además un concepto de energía que no se estaba explorando en la simulación. Welti, (2005) manifiesta que la interacción entre el aprendizaje de la temática de ondas, en especial la energía de 
la onda y el conocimiento previo sobre osciladores armónicos propicia la transferencia de los significados anteriores al nuevo fenómeno generando algunos errores. Sin embargo, en las respuestas del grupo experimental se evidencia que el error se puede generar en la dirección opuesta, es decir, el nuevo conocimiento sobre ondas se mezcla con el anterior sobre movimiento armónico simple.

Tabla 5: distribución de respuestas cuestionario de apoyo.

\begin{tabular}{|c|c|c|c|c|c|c|c|c|}
\hline \multicolumn{9}{|c|}{ Simulación 1: Elementos De Un Mas } \\
\hline \multicolumn{3}{|l|}{ Pregunta 1} & \multicolumn{3}{|l|}{ Pregunta 2} & \multicolumn{3}{|l|}{ Pregunta 3} \\
\hline $\begin{array}{l}\text { Cambia la velocidad } \\
\text { con que se mueve el } \\
\text { sistema masa-resorte. }\end{array}$ & 13 & $44,8 \%$ & $\begin{array}{l}\text { Aumenta o disminuye el } \\
\text { tamaño de la oscilación. }\end{array}$ & 10 & $34,5 \%$ & $\begin{array}{l}\text { A mayor frecuencia } \\
\text { mayor velocidad } y / 0 \\
\text { viceversa. }\end{array}$ & 16 & $55,2 \%$ \\
\hline $\begin{array}{l}\text { A mayor frecuencia } \\
\text { mayor velocidad } y / 0 \\
\text { viceversa. }\end{array}$ & 6 & $20,7 \%$ & $\begin{array}{l}\text { Cambia el tamaño de la } \\
\text { onda. }\end{array}$ & 6 & $20,7 \%$ & $\begin{array}{l}\text { Cambia la velocidad del } \\
\text { sistema masa-resorte. }\end{array}$ & 3 & $10,3 \%$ \\
\hline $\begin{array}{l}\text { El trazado se comprime } \\
\text { o expande } \\
\text { horizontalmente. }\end{array}$ & 5 & $17,2 \%$ & $\begin{array}{l}\text { Cambia la elongación de } \\
\text { la oscilación. }\end{array}$ & 6 & $20,7 \%$ & $\begin{array}{l}\text { Al modificar la velocidad } \\
\text { del resorte varía la } \\
\text { frecuencia. }\end{array}$ & 3 & $10,3 \%$ \\
\hline El periodo cambia. & 3 & $10,3 \%$ & Otras respuestas. & 7 & $24,1 \%$ & Otras respuestas. & 7 & $24,1 \%$ \\
\hline Otras respuestas. & 1 & $3,4 \%$ & & & & & & \\
\hline No responde. & 1 & $3,4 \%$ & & & & & & \\
\hline \multicolumn{3}{|l|}{ Pregunta 4} & \multicolumn{3}{|l|}{ Pregunta 5} & & & \\
\hline $\begin{array}{l}\text { A mayor frecuencia } \\
\text { menor periodo } y / 0 \\
\text { viceversa. }\end{array}$ & 19 & $65,5 \%$ & No tienen relación. & 14 & $48,3 \%$ & & & \\
\hline \multirow[t]{3}{*}{ Otras respuestas. } & 10 & $34,5 \%$ & $\begin{array}{l}\text { La frecuencia modifica la } \\
\text { velocidad y la amplitud } \\
\text { modifica el estiramiento }\end{array}$ & 5 & $17,2 \%$ & & & \\
\hline & & & $\begin{array}{l}\text { Frecuencia es la } \\
\text { cantidad de ondas por } \\
\text { minuto, amplitud es la } \\
\text { carga energética. }\end{array}$ & 5 & $17,2 \%$ & & & \\
\hline & & & Otras respuestas. & 5 & $17,2 \%$ & & & \\
\hline \multicolumn{9}{|c|}{ Simulación 2: Ecuaciones De Un Mas } \\
\hline \multicolumn{3}{|l|}{ Pregunta 1} & \multicolumn{3}{|c|}{ Pregunta 2} & \multicolumn{3}{|l|}{ Pregunta 3} \\
\hline $\begin{array}{l}\text { Cambia la posición de } \\
\text { inicio de la oscilación. }\end{array}$ & 10 & $34,5 \%$ & En el punto de equilibrio. & 13 & $44,8 \%$ & \begin{tabular}{|l|} 
En los puntos de \\
elongación.
\end{tabular} & 19 & $65,5 \%$ \\
\hline $\begin{array}{l}\text { Varía la oscilación y el } \\
\text { periodo de la onda. }\end{array}$ & 7 & $24,1 \%$ & $\begin{array}{l}\text { Cuando el ángulo vale } \\
\text { cero y la amplitud vale } \\
\text { cero. }\end{array}$ & 7 & $24,1 \%$ & $\begin{array}{l}\text { Cuando está a punto de } \\
\text { llegar a su máxima } \\
\text { amplitud. }\end{array}$ & 3 & $10,3 \%$ \\
\hline $\begin{array}{l}\text { Las gráficas empiezan } \\
\text { de un punto diferente. }\end{array}$ & 3 & $10,3 \%$ & $\begin{array}{l}\text { Cuando pasa por el } \\
\text { punto t. }\end{array}$ & 3 & $10,3 \%$ & Otras respuestas. & 6 & $20,7 \%$ \\
\hline \multirow[t]{2}{*}{ Otras respuestas. } & 9 & $31,0 \%$ & Otras respuestas. & 5 & $17,2 \%$ & No responde. & 1 & $3,5 \%$ \\
\hline & & & No responde. & 1 & $3,5 \%$ & & & \\
\hline \multicolumn{3}{|l|}{ Pregunta 4} & \multicolumn{3}{|l|}{ Pregunta 5} & \multicolumn{3}{|l|}{ Pregunta 6} \\
\hline $\begin{array}{l}\text { Por debajo del punto de } \\
\text { equilibrio. }\end{array}$ & 13 & $44,8 \%$ & En el punto de equilibrio. & 23 & $79,3 \%$ & $\begin{array}{l}\text { En los puntos de } \\
\text { elongación. }\end{array}$ & 9 & $31,0 \%$ \\
\hline $\begin{array}{l}\text { No tiene velocidad } \\
\text { negativa sino rapidez } \\
\text { negativa. }\end{array}$ & 7 & $24,1 \%$ & Otras respuestas. & 5 & $17,2 \%$ & En el punto de equilibrio. & 7 & $24,1 \%$ \\
\hline $\begin{array}{l}\text { Por debajo o encima } \\
\text { del punto de equilibrio. }\end{array}$ & 5 & $17,2 \%$ & No responde. & 1 & $3,5 \%$ & $\begin{array}{l}\text { En el punto de mayor } \\
\text { elongación. }\end{array}$ & 5 & $17,2 \%$ \\
\hline Otras respuestas. & 3 & $10,3 \%$ & & & & $\begin{array}{l}\text { Cuando se cumple una } \\
\text { oscilación. }\end{array}$ & 3 & $10,3 \%$ \\
\hline \multirow[t]{2}{*}{ No responde. } & 1 & $3,45 \%$ & & & & Otras respuestas. & 3 & $10,3 \%$ \\
\hline & & & & & & No responde. & 2 & $6,9 \%$ \\
\hline \multicolumn{9}{|c|}{ Simulación 3: Energía De Un Oscilador Armónico } \\
\hline \multicolumn{3}{|l|}{ Pregunta 1} & \multicolumn{3}{|c|}{ Pregunta 2} & \multicolumn{3}{|l|}{ Pregunta 3} \\
\hline $\begin{array}{l}\text { Depende de la } \\
\text { velocidad. }\end{array}$ & 7 & $24,1 \%$ & Depende de la posición. & 8 & $27,6 \%$ & $\begin{array}{l}\text { Porque es la suma de la } \\
\text { energía cinética y la } \\
\text { potencial. }\end{array}$ & 22 & $75,9 \%$ \\
\hline $\begin{array}{l}\text { Depende de la masa y } \\
\text { de la velocidad. }\end{array}$ & 7 & $24,1 \%$ & Depende de la altura. & 7 & $24,1 \%$ & Otras respuestas. & 3 & $10,3 \%$ \\
\hline
\end{tabular}


Tabla 5 (continuación)

\begin{tabular}{|c|c|c|c|c|c|c|c|c|}
\hline $\begin{array}{l}\text { Depende de la } \\
\text { elongación. }\end{array}$ & 5 & $17,2 \%$ & $\begin{array}{l}\text { De la elongación del } \\
\text { sistema masa-resorte. }\end{array}$ & 4 & $13,8 \%$ & No responde. & 4 & $13,8 \%$ \\
\hline Otras respuestas. & 5 & $17,2 \%$ & Otras respuestas. & 6 & $20,7 \%$ & & & \\
\hline No responde. & 5 & $17,2 \%$ & No responde. & 4 & $13,8 \%$ & & & \\
\hline \multicolumn{3}{|l|}{ Pregunta 4} & \multicolumn{3}{|l|}{ Pregunta 5} & & & \\
\hline $\begin{array}{l}\text { Vale cero en los puntos } \\
\text { de elongación y es } \\
\text { máxima en el punto de } \\
\text { equilibrio. }\end{array}$ & 16 & $55,2 \%$ & $\begin{array}{l}\text { Vale cero en el punto de } \\
\text { equilibrio y es máxima } \\
\text { en su elongación. }\end{array}$ & 16 & $55,2 \%$ & & & \\
\hline Otras respuestas. & 9 & $31,0 \%$ & Otras respuestas. & 7 & $24,1 \%$ & & & \\
\hline No responde. & 4 & $13,8 \%$ & No responde. & 6 & $20,7 \%$ & & & \\
\hline \multicolumn{9}{|c|}{ Simulación 4: Péndulo Simple } \\
\hline \multicolumn{3}{|l|}{ Pregunta 1} & \multicolumn{3}{|c|}{ Pregunta 2} & \multicolumn{3}{|l|}{ Pregunta 3} \\
\hline $\begin{array}{l}\text { A mayor longitud mayor } \\
\text { periodo y/o viceversa. }\end{array}$ & 19 & $65,5 \%$ & $\begin{array}{l}\text { A mayor gravedad } \\
\text { menor periodo. }\end{array}$ & 8 & $27,6 \%$ & El periodo no cambia. & 20 & $69,0 \%$ \\
\hline Otras respuestas. & 5 & $17,2 \%$ & Aumenta la velocidad. & 8 & $27,6 \%$ & Otras respuestas. & 3 & $10,3 \%$ \\
\hline \multirow[t]{3}{*}{ No responde. } & 5 & $17,2 \%$ & No responde. & 6 & $20,7 \%$ & No responde. & 6 & $20,7 \%$ \\
\hline & & & $\begin{array}{l}\text { Se mueve más rápido } \\
\text { disminuyendo el } \\
\text { periodo. }\end{array}$ & 3 & $10,3 \%$ & & & \\
\hline & & & Otras respuestas. & 4 & $13,8 \%$ & & & \\
\hline \multicolumn{9}{|c|}{ SIMULACIÓN 5: ELEMENTOS DE UNA ONDA } \\
\hline \multicolumn{3}{|l|}{ Pregunta 1} & \multicolumn{3}{|c|}{ Pregunta 2} & \multicolumn{3}{|l|}{ Pregunta 3} \\
\hline Cambia su velocidad. & 11 & $37,9 \%$ & Cambia su tamaño. & 9 & $31,0 \%$ & $\begin{array}{l}\text { Cambia la distancia entre } \\
\text { los puntos B y C. }\end{array}$ & 6 & $20,7 \%$ \\
\hline El trazado aumenta. & 4 & $13,8 \%$ & Se reduce la trayectoria. & 3 & $10,3 \%$ & $\begin{array}{l}\text { Cambia el recorrido } \\
\text { vertical }\end{array}$ & 4 & $13,8 \%$ \\
\hline Otras respuestas. & 3 & $10,3 \%$ & Otras respuestas. & 4 & $13,8 \%$ & $\begin{array}{l}\text { La onda se comprime o } \\
\text { se expande }\end{array}$ & 3 & $10,3 \%$ \\
\hline \multirow[t]{2}{*}{ No responde. } & 11 & $37,9 \%$ & No responde. & 13 & $44,8 \%$ & Otras respuestas & 3 & $10,3 \%$ \\
\hline & & & & & & No responde & 13 & $44,8 \%$ \\
\hline
\end{tabular}

\section{Paso 3. Test de Likert.}

Para cada ítem del test se calculó un valor promedio de acuerdo a la escala de valoración presentada en la Tabla 4. El promedio se calcula multiplicando el número de veces que aparece cada alternativa de respuesta (total desacuerdo, en desacuerdo, indeciso, de acuerdo o totalmente de acuerdo) por el valor asignado (1, 2, 3,4 o 5) a dicha alternativa (dependiendo si el ítem es favorable o desfavorable). Se realiza la suma de los productos mencionados y el resultado de la suma se divide entre el número de personas que dieron respuesta a ese ítem. Dado que el promedio así calculado puede generar un número que no es entero, se definió un rango de calificación para establecer una equivalencia cualitativa como se muestra en la Tabla 6.

Tabla 6: Rango de evaluación de resultados.

\begin{tabular}{|c|c|c|}
\hline \multirow{2}{*}{ Escala cualitativa } & Ítems favorables & Ítems desfavorables \\
\cline { 2 - 3 } & Rango de valor promedio & Rango de valor promedio \\
\hline Total Desacuerdo & 1 & $>4-5$ \\
\hline En desacuerdo & $>1-2$ & $>3-4$ \\
\hline Indeciso & $>2-3$ & $>2-3$ \\
\hline De acuerdo & $>3-4$ & $>1-2$ \\
\hline Totalmente de acuerdo & $>4-5$ & 1 \\
\hline
\end{tabular}

Tanto para los ítems favorables como los desfavorables, entre mayor sea el valor promedio, mayor será la fortaleza observada en el aspecto analizado para cada pregunta particular. Un ítem cuyo promedio esté entre 1 y 3 , representaría una situación adversa con respecto al aspecto que se está midiendo. Lo ideal es que los ítems favorables se encuentren en la escala cualitativa De acuerdo - Totalmente de acuerdo y que los ítems desfavorables se encuentren en la escala cualitativa Desacuerdo - Totalmente desacuerdo, es decir, en ambos casos un valor promedio mayor a 3 . Los resultados obtenidos se presentan en las Tablas 7 y 8 para el grupo control y en las Tablas 9 y 10 para el grupo experimental. 
Tabla 7: Resultados calificación cualitativa y cuantitativa - ítems favorables grupo control.

\begin{tabular}{|c|c|c|c|c|}
\hline \multirow{2}{*}{ No. Pregunta } & \multicolumn{4}{|c|}{ Calificación Promedio } \\
\cline { 2 - 5 } & \multicolumn{3}{|c|}{ Pre } & \multicolumn{2}{c|}{ Post } \\
\hline & Valor promedio & Escala cualitativa & Valor promedio & Escala cualitativa \\
\hline 1 & 3,6 & De acuerdo & 3,7 & De acuerdo \\
\hline 3 & 4,0 & De acuerdo & 3,8 & De acuerdo \\
\hline 5 & 3,5 & De acuerdo & 3,6 & De acuerdo \\
\hline 7 & 3,7 & De acuerdo & 3,8 & De acuerdo \\
\hline 9 & 4,1 & Totalmente de acuerdo & 4,0 & Totalmente de acuerdo \\
\hline
\end{tabular}

Tabla 8: Resultados calificación cualitativa y cuantitativa - ítems desfavorables grupo control.

\begin{tabular}{|c|c|c|c|c|}
\hline \multirow{2}{*}{ No. Pregunta } & \multicolumn{4}{|c|}{ Calificación Promedio } \\
\cline { 2 - 5 } & \multicolumn{3}{|c|}{ Pre } & Post \\
\hline & Valor promedio & Escala cualitativa & Valor promedio & Escala cualitativa \\
\hline 2 & 3,5 & En desacuerdo & 3,6 & En desacuerdo \\
\hline 4 & 3,8 & En desacuerdo & 3,7 & En desacuerdo \\
\hline 6 & 3,7 & En desacuerdo & 3,7 & En desacuerdo \\
\hline 8 & 4,1 & Totalmente desacuerdo & 4,2 & Totalmente desacuerdo \\
\hline 10 & 3,9 & En desacuerdo & 4,0 & En desacuerdo \\
\hline
\end{tabular}

Tabla 9: Resultados calificación cualitativa y cuantitativa - ítems favorables grupo experimental.

\begin{tabular}{|c|c|c|c|c|}
\hline \multirow{2}{*}{ No. Pregunta } & \multicolumn{4}{|c|}{ Calificación Promedio } \\
\cline { 2 - 5 } & \multicolumn{3}{|c|}{ Pre } & \multicolumn{2}{c|}{ Post } \\
\hline & Valor promedio & Escala cualitativa & Valor promedio & Escala cualitativa \\
\hline 1 & 4,4 & Totalmente de acuerdo & 4,5 & Totalmente de acuerdo \\
\hline 3 & 3,7 & De acuerdo & 3,9 & De acuerdo \\
\hline 5 & 3,9 & De acuerdo & 4,3 & Totalmente de acuerdo \\
\hline 7 & 4,0 & De acuerdo & 4,3 & Totalmente de acuerdo \\
\hline 9 & 4,1 & Totalmente de acuerdo & 4,2 & Totalmente de acuerdo \\
\hline
\end{tabular}

Tabla 10: Resultados calificación cualitativa y cuantitativa - ítems desfavorables grupo experimental.

\begin{tabular}{|c|c|c|c|c|}
\hline \multirow{2}{*}{ No. Pregunta } & \multicolumn{4}{|c|}{ Calificación Promedio } \\
\cline { 2 - 5 } & \multicolumn{3}{|c|}{ Pre } & Post \\
\hline & Valor promedio & Escala cualitativa & Valor promedio & Escala cualitativa \\
\hline 2 & 3,8 & En desacuerdo & 4,3 & Totalmente desacuerdo \\
\hline 4 & 3,8 & En desacuerdo & 3,9 & En desacuerdo \\
\hline 6 & 4,1 & Totalmente desacuerdo & 4,4 & Totalmente desacuerdo \\
\hline 8 & 4,7 & Totalmente desacuerdo & 4,7 & Totalmente desacuerdo \\
\hline 10 & 3,9 & En desacuerdo & 4,1 & Totalmente desacuerdo \\
\hline
\end{tabular}

Luego de la implementación de herramientas tecnológicas en los procesos de enseñanza de la física, los estudiantes reflejan mayor nivel de satisfacción con las nuevas metodologías de enseñanza implementadas, lo cual permitió mejorar los niveles de percepción de los estudiantes frente a la materia, esto para el caso del grupo experimental en comparación con el grupo control como se ve en la Figura 6.

La Tabla 11 muestra la variación porcentual entre el antes y después de la aplicación del test de Likert para el grupo control y el grupo experimental, como se puede observar el grupo experimental presentó mayor variación entre el antes y después de la aplicación del test, mientras que para el grupo control la variación no fue tan significativa, para algunos ítems incluso la variación fue negativa. 


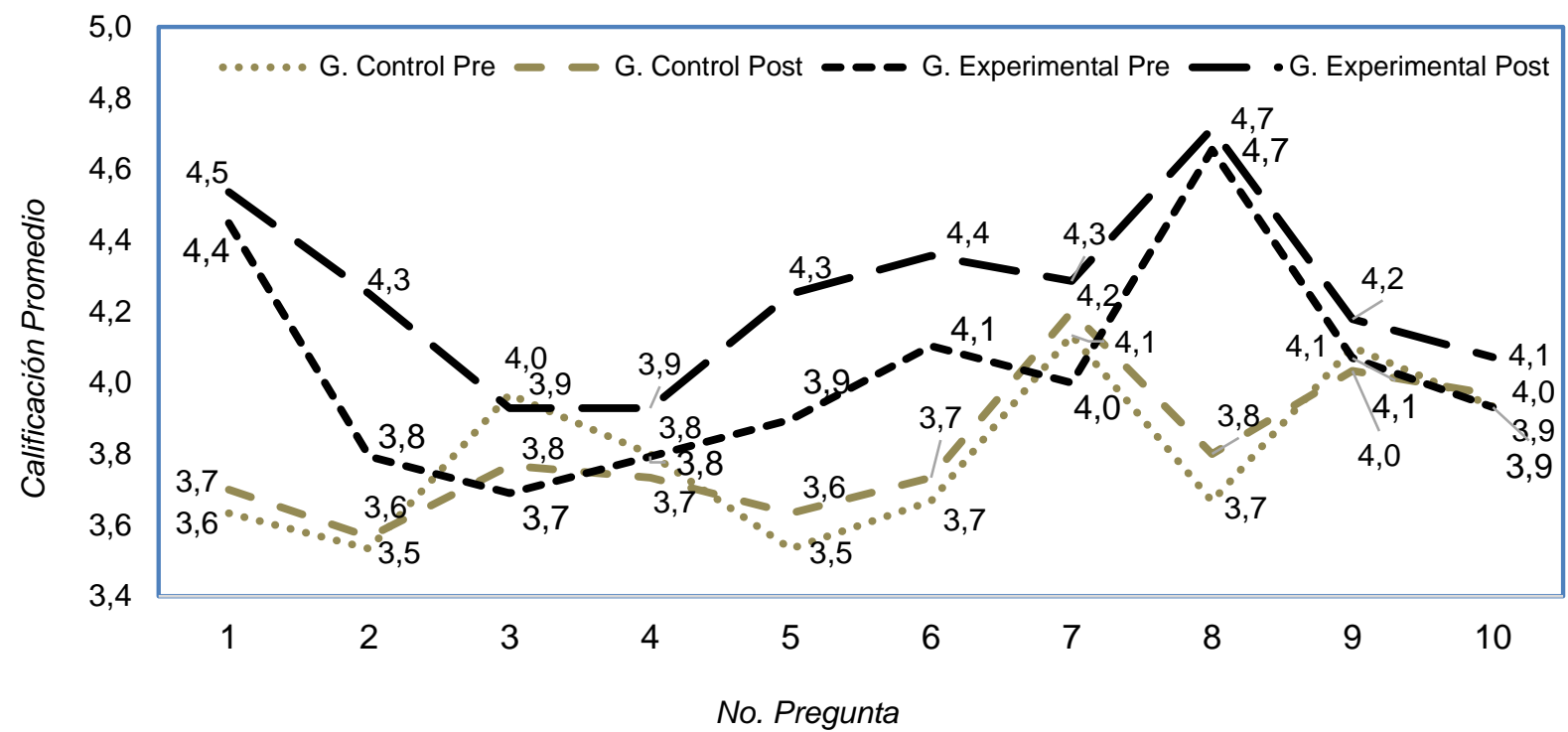

Fig. 6: Comparación calificación promedio de cada ítem antes y después del estudio para cada grupo.

Tabla 11: variación porcentual en la calificación promedio de los ítems del test de Likert.

\begin{tabular}{|c|c|c|}
\hline Pregunta & $\begin{array}{c}\text { Variación Porcentual } \\
\text { Grupo control }\end{array}$ & $\begin{array}{c}\text { Variación Porcentual } \\
\text { Grupo experimental }\end{array}$ \\
\hline 1 & $1,8 \%$ & $2,0 \%$ \\
\hline 2 & $0,9 \%$ & $12,0 \%$ \\
\hline 3 & $-5,0 \%$ & $6,5 \%$ \\
\hline 4 & $-1,8 \%$ & $3,6 \%$ \\
\hline 5 & $2,8 \%$ & $9,1 \%$ \\
\hline 6 & $1,8 \%$ & $6,2 \%$ \\
\hline 7 & $1,6 \%$ & $7,1 \%$ \\
\hline 8 & $3,6 \%$ & $1,3 \%$ \\
\hline 9 & $-1,6 \%$ & $2,7 \%$ \\
\hline 10 & $0,8 \%$ & $3,6 \%$ \\
\hline
\end{tabular}

\section{DISCUSIÓN Y ANÁLISIS FINAL DE LOS RESULTADOS}

A partir de los resultados se pudo evidenciar que existe una mejor respuesta de los estudiantes del grupo experimental, en comparación con el grupo control, a la disposición al aprendizaje práctico. El grupo experimental presentó un mayor nivel de satisfacción hacia la metodología de enseñanza de la asignatura, y a partir de los promedios obtenidos para los diferentes ítems del test de Likert se puede afirmar que los estudiantes de este grupo consideran que la metodología aplicada les permite explorar por su propia cuenta el comportamiento de los sistemas físicos estudiados, que los temas estudiados tienen aplicación práctica, prefieren aprender a través de contenidos multimedia más que con las clases tradicionales de tablero y marcador y les gustaría que otros docentes empleen herramientas similares a las utilizadas en esta asignatura. El grupo de control no tuvo cambios significativos con respecto a la evaluación realizada una vez terminó el periodo de evaluación.

Estudios como el realizado por (Dinescu et al., 2010) han mostrado que existen diferencias significativas en los grupos experimentales, observando mayor eficiencia en la participación activa e interactiva luego de la implementación de métodos de enseñanza didácticos. Otros estudios como (Martínez et al., 2018) reportan que un alto porcentaje de sus estudiantes consideran que la utilización de las TIC facilita su aprendizaje, de acuerdo a los resultados obtenidos en este estudio se evidencia que a la pregunta: "prefiero aprender a través de contenidos multimedia que con las clases tradicionales de tablero y marcador las respuestas dadas por los estudiantes del grupo experimental presentaron una variación positiva del 9,1\% y (Tapasco y Giraldo, 2017) informa que además estas aplicaciones son primordiales para los sistemas educativos actuales, a través de las cuales se puede lograr una mayor efectividad en el aprendizaje si se compara con los métodos de enseñanza tradicionales. 
Otras investigaciones como las hechas por (Tikhonova y Tereshkova, 2014) corroboran que los programas que hacen uso de herramientas tecnológicas mejoran su rendimiento en comparación con aquellos cursos formados a través de métodos de enseñanza habituales, en el alcance del presente estudio no se contempla evaluar la variación del rendimiento académico de los grupos, sin embargo, tal como lo muestran las respuestas a los cuestionarios de apoyo resumidas en la tabla 5 se logran tanto aciertos como desaciertos por parte de los estudiantes en la conceptualización de los temas estudiados. Investigaciones relacionadas indican que las plataformas interactivas, conocidas como entorno de aprendizaje virtual, son especialmente útiles cuando se enfocan en la enseñanza de ciencias y en particular de la física, la cual es generalmente de difícil comprensión para los estudiantes. (Martín y Serrano, 2009), al desarrollar su estudio observaron que los estudiantes que siguieron el curso con la ayuda de estas plataformas aumentaron su interés y por tanto obtuvieron mejores calificaciones en comparación con años anteriores para las materias objeto de ese análisis, en el presente estudio se evidenció un aumento en el interés de los estudiantes hacia la metodología de enseñanza, ante la pregunta: "me gustaría que otros docentes empleen herramientas similares a las utilizadas en esta asignatura" el grupo experimental muestra una variación positiva del 7,1\%.

Otros autores reconocen a través de sus estudios la importancia del papel de las TIC en la integración en las aulas escolares para promover la eficiencia en la enseñanza (Ezugwu et al., 2016) y mejorar las competencias de los estudiantes, llevando a mejores resultados en las áreas de aplicación (Ferraro, 2018). Además, estudios llevados a cabo por (Wang et al., 2018) demuestran que bajo un cambio en el modelo de enseñanza basado en tecnología, aplicado al modelado de la física, los maestros y estudiantes pueden iniciar el intercambio de aprendizaje en el aula de una forma más participativa, de manera que se establezcan conceptos y conocimientos correctos de las diferentes temáticas y además mejore el proceso cognitivo de los estudiantes, ofreciendo un enfoque científico y mejorando su razonamiento. Este estudio concluyó que los estudiantes que se sometieron a las nuevas metodologías de enseñanza en el aula, tuvieron una mejora notable en cuanto a los conceptos aplicados a los modelos de física. No hay que desconocer que algunos de los mayores obstáculos que se presentan en la implementación adecuada de las nuevas metodologías de enseñanza basadas en TIC en algunas regiones, son entre otros la limitante de la conectividad a internet y además los pocos recursos en cuanto a la disponibilidad de computadoras, acceso a energía eléctrica y otros accesorios requeridos (Ezugwu et al., 2016). Es importante entonces que para la implementación de las nuevas metodologías de enseñanza, exista alto compromiso del gobierno y de las instituciones sobre los recursos otorgados para una correcta aplicación en las aulas. Además es de vital importancia el tiempo que los maestros dediquen en la cátedra a estos nuevos modelos y además el entrenamiento y compromiso que estos tengan (Claro et al., 2018).

Entonces a partir de la implementación de una metodología de enseñanza asistida por simulaciones computarizadas, se logró aumentar el nivel de satisfacción del estudiante frente al aprendizaje de la física. Si bien es cierto existe una amplia oferta de software orientado a la simulación de fenómenos físicos, geogebra ofrece versatilidad al docente a la hora de crear las simulaciones, permitiendo personalizar las mismas a través de la creación de escenarios específicos al contexto del estudiante, mientras que los paquetes de software existentes funcionan con escenarios predeterminados. Como líneas de investigación futura se plantean: incluir a los estudiantes en el proceso de modelado de los las simulaciones de fenómenos físicos. Estudiar el impacto de la metodología en otras áreas del conocimiento e incluir el uso de dispositivos portátiles tales como teléfonos inteligentes y tabletas.

\section{CONCLUSIONES}

A partir de los resultados obtenidos, se pueden extraer las siguientes conclusiones: 1) La metodología de enseñanza tradicional aplicada en el grupo control no favorece por si sola un cambio significativo en el nivel de satisfacción del estudiante frente a la enseñanza de la física. 2) Existe una respuesta positiva de los estudiantes del grupo experimental a la implementación de la metodología, durante el periodo de estudio evaluado. 3) bajo las condiciones del estudio llevado a cabo, los estudiantes del grupo experimental prefieren aprender a través de contenidos multimedia más que con las clases tradicionales y además consideran que este tipo de herramientas podrían ser empleadas en otras asignaturas para facilitar la comprensión de la temática orientada. 4) La calificación promedio del nivel de satisfacción para cada ítem del test de Likert presentó una variación porcentual más significativa en el grupo experimental en comparación con el grupo control.

\section{AGRADECIMIENTOS}

Los resultados que presenta este artículo hacen parte de la tesis de maestría titulada: "Diseño e implementación de una metodología para el proceso de enseñanza aprendizaje de la física asistido por simulación para mejorar el grado de satisfacción de los estudiantes hacia el aprendizaje" del programa Maestría en Enseñanza de las Ciencias Exactas y Naturales de la U. Nacional de Colombia sede Manizales. 


\section{REFERENCIAS}

Caligaris, M.G., M.E. Schivo y M.R. Romiti, Calculus \& GeoGebra, an Interesting Partnership, doi:10.1016/j.sbspro.2015.01.735, Procedia - Social and Behavioral Sciences, 174, 1183-1188 (2015)

Cardona, M.E. y S. López, Una Revisión de Literatura Sobre el Uso de Sistemas de Adquisición de Datos Para la Enseñanza de la Física en la Educación Básica, Media y en la Formación de Profesores, doi:10.1590/1806-9126-rbef2016-0308, Revista Brasileira de Ensino de Física, 39(4), e4404-1 - e4404-11 (2017)

Carpenter, Y., E.B. Moore y K.K. Perkins, ConfChem Conference on Interactive Visualizations for Chemistry Teaching and Learning: Using an Interactive Simulation to Support Development of Expert Practices for Balancing Chemical Equations, doi:10.1021/acs.jchemed.5b00546, Journal of Chemical Education, 93(6), 1150-1151 (2016)

Chang, K., Y. Chen, H. Lin e Y. Sung, Effects of Learning Support in Simulation-Based Physics Learning, doi:10.1016/j.compedu.2008.01.007, Computers and Education, 51(4), 1486-1498 (2008)

Claro, M., A. Salinas y otros cinco autores, Teaching in a Digital Environment (TIDE): Defining and Measuring Teachers' Capacity to Develop Students' Digital Information and Communication Skills, doi:10.1016/j.compedu.2018.03.001, Computers and Education, 121, 162-174 (2018)

Dega, B. G., J. Kriek y T. F. Mogese, Students' Conceptual Change in Electricity and Magnetism Using Simulations: A Comparison of Cognitive Perturbation and Cognitive Conflict, doi:10.1002/tea.21096, Journal of Research in Science Teaching, 50, 677-698 (2013)

Delgadillo, F. A., Propuesta Didáctica para la Enseñanza de la Hidrostática en Ciclo 5 a Partir de Situaciones Paradójicas; Tesis de Maestría, Universidad Nacional de Colombia, Facultad de Ciencias, Bogotá, Colombia (2013)

Díez, J. A., Manual de Herramientas Pedagógicas para la Enseñanza de Técnicas Instrumentales de Análisis Químico y Físico, Tesis de Maestría, Universidad Nacional de Colombia, Facultad de Ciencias, Medellín, Colombia (2015)

Dinescu, L., M. Dinica y C. Miron, Active Strategies - Option and Necessity for Teaching Science in Secondary and High School Education, doi:10.1016/j.sbspro.2010.03.579, Procedia - Social and Behavioral Sciences, 2(2), 3724-3730 (2010)

Ezugwu, A.E., P.O. Ofem y otros tres autores, An Empirical Evaluation of the Role of Information and Communication Technology in Advancement of Teaching and Learning, doi:10.1016/j.procs.2016.07.384, Procedia Computer Science, 92, 568-577 (2016)

Ferraro, S., Is Information and Communication Technology Satisfying Educational Needs at School? doi:10.1016/j.compedu.2018.04.002, Computers and Education, 122, 194-204 (2018)

Harpe, S.E., How to Analyze Likert and other Rating Scale Data, doi:10.1016/j.cptl.2015.08.001, Currents in Pharmacy Teaching and Learning, 7(6), 836-850 (2015)

Hartley, J., Some Thoughts on Likert-type Scales, doi:10.1016/S1697-2600(14)70040-7, International Journal of Clinical and Health Psychology, 14 (1), 83-86 (2013)

Huapaya, C.R., G.M. Arona y F.A. Lizarralde, Enseñanza de la Ingeniería con Sistemas Tutoriales Inteligentes, doi:10.4067/S0718-07642005000500012, Información Tecnológica, 16(5), 75-78 (2005)

Jaramillo, G.C., Elaboración e Implementación de una Herramienta Didáctica que Permita Enseñar la Lógica Proposicional a los Estudiantes de Grado Sexto, Tesis de Maestría, Universidad Nacional de Colombia, Facultad de Ciencias Exactas y Naturales, Manizales, Colombia (2016)

Jaramillo, G. J., Diseño de un Objeto Virtual para la Enseñanza-Aprendizaje de los Conceptos Matemáticos de Nivelación Previos al Estudio de la Química General, Tesis de Maestría, Universidad Nacional de Colombia, Facultad de Ciencias Exactas y Naturales, Manizales, Colombia (2016)

Krobthong, T., Teaching University Physics by Using Interactive Science Simulations Methods, doi:10.1016/j.sbspro.2015.07.240, Procedia - Social and Behavioral Sciences, 197, 1811-1817 (2015)

León, C.E., El Pensamiento Covariacional y GeoGebra: Herramientas para la Explicación Científica de Algunas Realidades, doi:10.17227/01203916.6969, Tecné Episteme y Didaxis TED, 42, 159-171 (2017)

Lopez, S., E.A. Veit e I. S. Araujo, Una Revisión de Literatura Sobre el Uso de Modelación y Simulación Computacional para la Enseñanza de la Física en la Educación Básica y Media, doi: 10.1590/1806-9126-RBEF-2015-0031, Revista Brasileira de Ensino de Física, 38(2), e2401-1-e2401-16 (2016)

Márquez, D. A. y O. O. Cárdenas, Implementación de un Laboratorio Virtual para la Enseñanza de Controladores PID, doi:10.1612/inf.tecnol.3884it.07, Información Tecnológica, 19(3), 75-78 (2008)

Martín, T. y A. Serrano, The Role of New Technologies in the Learning Process: Moodle as a Teaching Tool in Physics, doi:10.1016/j.compedu.2008.06.005, Computers and Education, 52(1), 35-44 (2009)

Martínez, L. D., F.J. Hinojo e I.A. Díaz, Aplicación de las Tecnologías de la Información y la Comunicación (TIC) en los Procesos de Enseñanza- Aprendizaje por parte de los Profesores de Química, doi:10.4067/S0718-07642018000200041, Información Tecnológica, 29(2), 41-52 (2018)

Rossi, A. P. y P. N. Baldini, Herramienta Computacional para la Enseñanza de la Evaluación del Campo Eléctrico en Instalaciones Industriales, doi:10.1612/inf.tecnol.3921it.07, Información Tecnológica, 19(3), 79-88 (2008) 
Rubio, O. E., Los Mapas Conceptuales como Estrategia para la Enseñanza - Aprendizaje de los Gases; Tesis de Maestría, Universidad Nacional de Colombia, Facultad de Ciencias Exactas y Naturales, Manizales, Colombia (2013)

Smetana, L. K. y R. L. Bell, Computer Simulations to Support Science Instruction and Learning: A Critical Review of the Literature, doi:10.1080/09500693.2011.605182, International Journal of Science Education, 34(9), 1337-1370 (2012)

Stoica, D., F. Paragina y otros tres autores, The Interactive Whiteboard and the Instructional Design in Teaching Physics, doi:10.1016/j.sbspro.2011.04.292, Procedia - Social and Behavioral Sciences, 15, 3316-3321 (2011)

Summak, M. S., M. Bağlibel y M. Samancioğlu, Technology Readiness of Primary School Teachers: A Case Study in Turkey, doi:10.1016/j.sbspro.2010.03.393, Procedia - Social and Behavioral Sciences, 2(2), 2671-2675 (2010)

Tapasco, O. A. y J. A. Giraldo, Estudio Comparativo sobre Percepción y Uso de las TIC entre Profesores de Universidades Públicas y Privadas, doi:10.4067/S0718-50062017000200002, Formación Universitaria, 10(2), 3-12 (2017)

Tikhonova, E.V. y N.S. Tereshkova, Information and Communication Technologies in the Teaching of Interpreting, doi:10.1016/j.sbspro.2014.10.181, Procedia - Social and Behavioral Sciences, 154, 534-538 (2014)

Wang, J., M. Jou, Y. Lv y C. Huang, An Investigation on Teaching Performances of Model-Based Flipping Classroom for Physics Supported by Modern Teaching Technologies, doi:10.1016/j.chb.2018.02.018, Computers in Human Behavior, 84, 36-48 (2018)

Welti, R., Obstáculos Conceptuales en el Aprendizaje de la Energía de las Ondas, doi:10.1590/S180611172005000300027, Rev. Bras. Ensino Fís., 27(3), 1806-1117 (2005)

Zacharia, Z. C., Comparing and Combining Real and Virtual Experimentation: An Effort to Enhance Students' Conceptual Understanding of Electric Circuits, doi:10.1111/j.1365-2729.2006.00215.x, Journal of Computer Assisted Learning, 23(2), 120-132 (2007)

Zucker, A. y S. T. Hug, Teaching and Learning Physics in a 1:1 Laptop School, doi:10.1007/s10956-008-9125-3, Journal of Science Education and Technology, 17(6), 586-594 (2008)

Zuluaga, C. A., Diseño e Implementación de un Curso Virtual para la Enseñanza-Aprendizaje del Movimiento de los Cuerpos en dos Dimensiones Mediante el uso de Unidades Didácticas: Estudio de Caso en el Grado $10^{\circ}$ de la Institución Educativa San Jorge, de la Ciudad de Manizales, Tesis de Maestría, Universidad Nacional de Colombia, Facultad de Ciencias Exactas y Naturales, Manizales, Colombia (2016) 
